\title{
Gestation-specific reference intervals for comprehensive spot urinary steroid hormone metabolite analysis in normal singleton pregnancy and 6 weeks postpartum
}

\author{
Hiten D. Mistry ${ }^{1}$, Nicole Eisele ${ }^{1}$, Geneviève Escher ${ }^{1}$, Bernhard Dick ${ }^{1}$, Daniel Surbek² ${ }^{2}$ Christian Delles ${ }^{3}$, \\ Gemma Currie ${ }^{3}$, Dietmar Schlembach ${ }^{4}$, Markus G. Mohaupt ${ }^{1,5^{*}}$ and Carine Gennari-Moser ${ }^{1+}$
}

\begin{abstract}
Background: Normal pregnancy depends on pronounced adaptations in steroid hormone concentrations. Although in recent years, the understanding of these hormones in pregnancy has improved, the interpretation is hampered by insufficient reference values. Our aim was to establish gestation-specific reference intervals for spot urinary steroid hormone levels in normal singleton pregnancies and 6 weeks postpartum.

Methods: Cross-sectional multicentre observational study. Women recruited between 2008 and 2013 at 3 University Hospitals in Switzerland (Bern), Scotland (Glasgow) and Austria (Graz). Spot urine was collected from healthy women undergoing a normal pregnancy (age, 16-45 years; mean, 31 years) attending routine antenatal clinics at gestation weeks 11,20 , and 28 and approximately 6 weeks postpartum. Urine steroid hormone levels were analysed using gas-chromatography mass spectrometry. Creatinine was also measured by routine analysis and used for normalisation.

Results: From the results, a reference interval was calculated for each hormone metabolite at each trimester and 6 weeks postpartum. Changes in these concentrations between trimesters and postpartum were also observed for several steroid hormones and followed changes proposed for index steroid hormones.

Conclusions: Normal gestation-specific reference values for spot urinary steroid hormones throughout pregnancy and early postpartum are now available to facilitate clinical management and research approaches to steroid hormone metabolism in pregnancy and the early postpartum period.
\end{abstract}

Keywords: Pregnancy reference values, Urine, Steroid metabolites

\section{Background}

A successful healthy pregnancy is recognised by pregnancy-related changes in hormone concentrations, characterised by elevated levels of several circulating steroid hormones, which normally increase as pregnancy progresses $[1,2]$. Changes in maternal hormone

\footnotetext{
* Correspondence: markus.mohaupt@insel.ch

${ }^{\dagger}$ Equal contributors

'Department of Nephrology, Hypertension, Clinical Pharmacology and Clinical Research, University of Bern, 3010 Berne, Switzerland

${ }^{5}$ Division of Hypertension, Department of Nephrology, Hypertension, Clinical Pharmacology and Clinical Research, University of Bern, CH-3010 Berne, Switzerland

Full list of author information is available at the end of the article
}

concentrations play a critical role in modulating the metabolic and immunological changes required for successful pregnancy outcome; they also have an important counterpart as the fetoplacental unit develops. There is interest in how endogenous steroid hormones and their respective metabolites have influence or are altered in studies during pregnancy with respect to fetal size, preterm birth, multiple pregnancies, regulation of partition, hypertensive disorders of pregnancy and other conditions [3-5]. Many of these previously published studies on steroid metabolite concentrations have produced inconsistent findings, mainly due to technological issues or lack of specificity. However, the development of

\section{Ciomed Central}

(c) 2015 Mistry et al. Open Access This article is distributed under the terms of the Creative Commons Attribution 4.0 International License (http://creativecommons.org/licenses/by/4.0/), which permits unrestricted use, distribution, and reproduction in any medium, provided you give appropriate credit to the original author(s) and the source, provide a link to the Creative Commons license, and indicate if changes were made. The Creative Commons Public Domain Dedication waiver (http://creativecommons.org/publicdomain/zero/1.0/) applies to the data made available in this article, unless otherwise stated. 
sensitive mass spectrometry based assays that can accurately measure individual steroid hormone concentrations [6], allows improvement of our understanding of gestation-dependent trends of these hormones. As many conventional assays such as radioimmuno assays (RIA) or enzyme-linked immunosorbent assays (ELISA) are compromised by significant cross-reactivity and lack of specificity, future analyses will require mass spectrometry or other more specific techniques [7]. Earlier studies using GC-MS-based techniques in pregnancy reported analyses in different biological fluids, such as in plasma samples [8], in low numbers of pregnant women [9], in disease states $[10,11]$ and providing only partial steroid hormone panels [9].

Physiological changes in pregnancy, including $50 \%$ plasma volume expansion or significant changes in binding protein concentrations, mean that plasma levels and non-pregnant urinary steroid hormone reference intervals may not be appropriate in pregnancy [12]. Although in recent years, our understanding of the role of these hormones in pregnancy has improved, the interpretation of these levels is still difficult. We are not aware of any previous work reporting spot urinary steroid metabolite profiles during pregnancy, calculating reference intervals recommended for cross-sectional data that varies with gestational age $[13,14]$.

Given the importance of changes in steroid hormones during pregnancy and that reliable, clinically and scientifically useful reference intervals are not available for urine; we set out to determine gestation-specific reference intervals in a cross-sectional study of women during normal pregnancy and 6 weeks postpartum. Furthermore, due to the problems associated with collection of $24 \mathrm{~h}$ urine, especially during pregnancy, an advantage of this study was to produce reference values using spot urines from 3 different populations.

\section{Materials and methods Subjects}

A first set of cross-sectional healthy pregnant women recruited to the Bernese pregnancy registry were included in the study. Visits were at week $11 \pm 2(n=25), 20 \pm 2$ $(n=32), 28 \pm 2(n=26)$ and approximately 6 weeks postpartum $(n=40)$. The study was approved by the ethics committee of the Canton of Berne, Switzerland.

A second set of samples were derived from a large prospective pregnancy cohort from the Queen Mother's, Princess Royal Maternity and Southern General Hospitals in Glasgow, Scotland. First trimester samples $(11 \pm$ 2 weeks' gestation; $n=46$ ) were taken at booking. The study was approved by the ethics committee of the West of Scotland Research Ethics Committee.

The third cohort of samples consisted of women from Graz, Austria. Cross-sectional samples were obtained from each trimester (weeks $11 \pm 2(n=10), 20 \pm 2(n=8)$ and $28 \pm 2(n=14))$ following ethical approval from the University of Graz, Austria.

All study subjects included from the 3 cohorts were in keeping with the declaration of Helsinki and all participants provided written, informed consent. Only those women maintaining a regular, normotensive, uncomplicated pregnancy without gestational diabetes, hepatic, renal or other obvious diseases with no fetal abnormalities such as fetal growth restriction were included in this study. Clinical data were prospectively collected and either existing hospital files or the woman's personal history reviewed to exclude any pre-existing disease, including pregnancy outcome. In total, 81 samples were used from the first trimester and 40 for all other trimesters and postpartum.

\section{Urine sampling}

Morning fasting urine samples were obtained from pregnant women as has been described for population-based assessments [15]. In all urine collections, sodium and creatinine concentrations were determined using routine methods. Urine aliquots were stored at $-80{ }^{\circ} \mathrm{C}$ until further analysis.

\section{Assessment of urinary steroid hormones by GC-MS}

Steroid hormone measurements for samples from all 3 cohorts were conducted in Bern using the same GC-MS method, as described earlier $[6,7,16]$. In short, preparation consisted of pre-extraction, enzymatic hydrolysis, extraction from the hydrolysis mixture, derivatisation and gel filtration. All solvents were purchased from Merck, USA). Medroxyprogesterone (2.5 $\mu$ g; Steraloids, Inc. Switzerland) was added as recovery standard to $1.5 \mathrm{ml}$ urine. The sample was extracted on a Sep Pak C18 column (Waters, Switzerland), dried, reconstituted in $0.1 \mathrm{M}$ acetate buffer ( $\mathrm{pH} 4.6$ ) and hydrolyzed with powdered Helix pomatia enzyme (12.5 mg, Sigma-Aldrich, $\mathrm{UK})$ and $12.5 \mu \mathrm{l}$ of $\beta$-glucuronidase/arylsulfatase liquid enzyme (Roche, USA) at $55{ }^{\circ} \mathrm{C}$ for $3 \mathrm{~h}$. The resulting free steroids were extracted on a Sep Pak C18 cartridge and $2.5 \mu \mathrm{g}$ stigmasterol (Steraloids, Inc. Switzerland) plus $0.15 \mu \mathrm{g}$ of $3 \beta 5 \beta$-TH-Aldo (custom-made by Taros Chemicals, Germany) were added as a standard for derivatisation

Table 1 Basic demographic and pregnancy data from there cohorts used in the study

\begin{tabular}{llll}
\hline Parameter & Bern & Glasgow & Graz \\
\hline Maternal Age $(\mathrm{yrs})$ & $31.5 \pm 3.8$ & $29.6 \pm 6.3$ & $31.8 \pm 5.3$ \\
Booking BMl $\left(\mathrm{kg} / \mathrm{m}^{2}\right)$ & $25.5 \pm 3.8$ & $24.3 \pm 5.4$ & $23.8 \pm 3.7$ \\
Gestational age at delivery $(\mathrm{Wks})$ & $39 \pm 1.2$ & $40 \pm 1.2$ & $39 \pm 1.3$ \\
Birthweight (g) & $3505 \pm 658$ & $3477 \pm 530$ & $3304 \pm 346$ \\
Baby gender (\% male) & 51 & 46 & 40 \\
\hline
\end{tabular}


Table 2 Steroid hormone metabolite[ $\mu \mathrm{g} / \mathrm{mmol}$ urinary creatinine]

\begin{tabular}{|c|c|c|c|c|c|c|c|c|c|c|c|c|}
\hline \multirow[b]{3}{*}{ move to a separate row } & \multicolumn{4}{|c|}{$1^{\text {st }}$ trimester $(n=81)$} & \multicolumn{4}{|c|}{$2^{\text {nd }}$ trimester $(n=40)$} & \multicolumn{4}{|c|}{$3^{\text {rd }}$ trimester $(n=40)$} \\
\hline & \multirow[b]{2}{*}{$\begin{array}{l}\text { Mean } \pm \\
\text { SD }\end{array}$} & \multirow[b]{2}{*}{ Median } & \multicolumn{2}{|c|}{ Reference intervals } & \multirow[b]{2}{*}{$\begin{array}{l}\text { Mean } \pm \\
\text { SD }\end{array}$} & \multirow[b]{2}{*}{ Median } & \multicolumn{2}{|c|}{ Reference intervals } & \multirow[b]{2}{*}{$\begin{array}{l}\text { Mean } \pm \\
\text { SD }\end{array}$} & \multirow[b]{2}{*}{ Median } & \multicolumn{2}{|c|}{ Reference intervals } \\
\hline & & & $\begin{array}{l}2.5^{\text {th }} \text { percentile } \\
{[90 \% \mathrm{Cl}]}\end{array}$ & $\begin{array}{l}97.5^{\text {th }} \text { percentile } \\
{[90 \% \mathrm{Cl}]}\end{array}$ & & & $\begin{array}{l}2.5^{\text {th }} \text { percentile } \\
{[90 \% \mathrm{Cl}]}\end{array}$ & $\begin{array}{l}97.5^{\text {th }} \text { percentile } \\
{[90 \% \mathrm{Cl}]}\end{array}$ & & & $\begin{array}{l}2.5^{\text {th }} \text { percentile } \\
{[90 \% \mathrm{Cl}]}\end{array}$ & $\begin{array}{l}97.5^{\text {th }} \text { percentile } \\
{[90 \% \mathrm{Cl}]}\end{array}$ \\
\hline Androsterone & $322 \pm 196$ & 289 & $40.0[36.0,91.7]$ & $764[740,780]$ & $209 \pm 172$ & 159 & $17.3[16.6,55.5]$ & $746[665,748]$ & $237 \pm 274$ & 134 & $5.9[5.5,38.0]$ & $1409[844,1423]$ \\
\hline Etiocholanolone & $231 \pm 141$ & 211 & $31.0[30.9,65.3]$ & $617[542,688]$ & $149 \pm 95.4$ & 126 & $24.0[23.7,61.5]$ & $518[355,522]$ & $163 \pm 176$ & 108 & $6.5[6.0,34.9]$ & $774[707,776]$ \\
\hline 11-Oxo-Etiocholanolon & $38.6 \pm 18.7$ & 37.6 & $6.3[1.7,11.5]$ & $84.4[74.1,87.0]$ & $38.0 \pm 19.0$ & 35.1 & $6.4[6.3,15.3]$ & $77.8[72.7,77.9]$ & $49.1 \pm 52.3$ & 36.7 & $5.8[5.8,13.5]$ & $325[125,325]$ \\
\hline $11 \beta$-Hydroxy-Androsterone & $89.8 \pm 53.3$ & 75.1 & $20.8[17.1,23.1]$ & $233[212,234]$ & $70.8 \pm 44.3$ & 62.6 & $7.3[7.1,21.2]$ & $192[188,193]$ & $94.3 \pm 123$ & 58.7 & $4.9[4.5,21.4]$ & $716[315,726]$ \\
\hline $\begin{array}{l}11 \beta \text {-Hydroxy- } \\
\text { Etiocholanolon }\end{array}$ & $31.5 \pm 22.1$ & 29.3 & $5.7[4.8,8.6]$ & $83.8[65.3,163]$ & $39.5 \pm 28.6$ & 28.6 & $3.2[3.1,11.6]$ & $112[97.7,112]$ & $43.4 \pm 28.8$ & 37.0 & $10.6[10.5,12.7]$ & $141[105,141]$ \\
\hline Dehydroepiandrosterone & $86.2 \pm 158$ & 23.1 & $1.5[1.5,4.4]$ & $759[569,774]$ & $49.6 \pm 87.5$ & 18.4 & $0.7[0.7,2.1]$ & $380[348,380]$ & $61.6 \pm 167$ & 12.0 & $1.3[1.3,2.5]$ & $919[520,929]$ \\
\hline 5-Androstene-3 $\beta, 17 \beta$-diol & $26.8 \pm 25.6$ & 18.8 & $1.4[0.9,4.1]$ & $125[78.0,144]$ & $20.1 \pm 16.1$ & 16.0 & $0.9[0.9,5.1]$ & $85.3[55.2,86]$ & $25.5 \pm 29.8$ & 17.9 & $1.0[1.0,5.5]$ & $175[69.0,175]$ \\
\hline 5-Androstene-3 $\beta, 17 \beta$-triol & $17.3 \pm 57.2$ & 5.3 & $0.7[0.7,1.8]$ & $225[92.6,454]$ & $10.9 \pm 33.0$ & 2.6 & $0.8[0.8,1.0]$ & $164[139,165]$ & $9.9 \pm 20.8$ & 2.6 & $0.7[0.7,0.9]$ & $115[51.5,115]$ \\
\hline $\begin{array}{l}\text { 16a-Hydroxy- } \\
\text { dehydroepiandrostrerone }\end{array}$ & $96.4 \pm 101$ & 60.7 & $2.9[1.2,14.4]$ & $415[277,600]$ & $107 \pm 107$ & 88.7 & $2.1[2.0,12.5]$ & $569[259,577]$ & $154 \pm 340$ & 77.5 & $3.4[3.3,13.0]$ & $2139[376,2139]$ \\
\hline $\begin{array}{l}\text { 5-Androstene-3 } \beta, 16 a, 17 \beta- \\
\text { triol }\end{array}$ & $84.3 \pm 75.0$ & 55.8 & $9.2[7.5,22.2]$ & $278[209,306]$ & $65.4 \pm 60.0$ & 52.8 & $11.7[11.7,27.7]$ & $313[127,317]$ & $107 \pm 155$ & 60.2 & $12.4[12.3,18.5]$ & $868[454,868]$ \\
\hline $\begin{array}{l}\text { 5-Pregnene-3 } \beta, 16 a, 17,20 a- \\
\text { triol }\end{array}$ & $48.2 \pm 56.5$ & 36.8 & $1.2[0.9,3.5]$ & $317[161,397]$ & $20.6 \pm 25.5$ & 13.6 & $0.9[0.9,2.1]$ & $151[50.7,154]$ & $38.1 \pm 111$ & 11.4 & $1.7[1.7,2.9]$ & $690[107,690]$ \\
\hline Testosterone & $2.0 \pm 1.8$ & 1.6 & $0.3[0.2,0.5]$ & $9.4[6,11.1]$ & $2.4 \pm 3.2$ & 1.3 & $0.3[0.3,0.4]$ & $18.5[7.4,18.8]$ & $3.6 \pm 5.6$ & 2.0 & $0.5[0.5,0.7]$ & $27.8[27.8,28.1]$ \\
\hline 5a-Dihydrotestosterone & $4.6 \pm 3.2$ & 3.6 & $0.6[0.5,1.1]$ & $12.3[11.4,12.9]$ & $3.7 \pm 2.6$ & 3.2 & $0.5[0.5,1.2]$ & $11.1[10.1,11.1]$ & $5.7 \pm 3.7$ & 4.6 & $0.4[0.4,1.7]$ & $19.1[11.3,19.3]$ \\
\hline
\end{tabular}


Table 3 Steroid hormone metabolite $[\mu \mathrm{g} / \mathrm{mmol}$ urinary creatinine]

\begin{tabular}{|c|c|c|c|c|c|c|c|c|c|c|c|c|}
\hline \multirow[b]{3}{*}{ move to a separate row } & \multicolumn{4}{|c|}{$1^{\text {st }}$ trimester $(n=81)$} & \multicolumn{4}{|c|}{$2^{\text {nd }}$ trimester $(n=40)$} & \multicolumn{4}{|c|}{$3^{\text {rd }}$ trimester $(n=40)$} \\
\hline & \multirow[b]{2}{*}{ Mean \pm SD } & \multirow[b]{2}{*}{ Median } & \multicolumn{2}{|c|}{ Reference intervals } & \multirow[b]{2}{*}{ Mean \pm SD } & \multirow[b]{2}{*}{ Median } & \multicolumn{2}{|c|}{ Reference intervals } & \multirow[b]{2}{*}{ Mean \pm SD } & \multirow[b]{2}{*}{ Median } & \multicolumn{2}{|c|}{ Reference intervals } \\
\hline & & & $\begin{array}{l}2.5^{\text {th }} \\
\text { percentile } \\
{[90 \% \mathrm{Cl}]}\end{array}$ & $\begin{array}{l}97.5^{\text {th }} \\
\text { percentile } \\
{[90 \% \mathrm{Cl}]}\end{array}$ & & & $\begin{array}{l}2.5^{\text {th }} \text { percentile } \\
{[90 \% \mathrm{Cl}]}\end{array}$ & $\begin{array}{l}97.5^{\text {th }} \\
\text { percentile } \\
{[90 \% \mathrm{Cl}]}\end{array}$ & & & $\begin{array}{l}2.5^{\text {th }} \\
\text { percentile } \\
{[90 \% \mathrm{Cl}]}\end{array}$ & $\begin{array}{l}97.5^{\text {th }} \\
\text { percentile } \\
{[90 \% \mathrm{Cl}]}\end{array}$ \\
\hline Estriol & $135 \pm 151$ & 79.9 & $10.6[3.5,17.0]$ & $545[489,901]$ & $975 \pm 584$ & 836 & $143[143,2881]$ & $2877[2660,2881]$ & $2651 \pm 2651$ & 1955 & $636[636,764]$ & $\begin{array}{c}15192 \\
{[9171,15116]}\end{array}$ \\
\hline $17 \beta$-Estradiol & $9.1 \pm 6.7$ & 7.3 & $0.7[0.6,2.1]$ & $26.4[24.8,28.8]$ & $16.6 \pm 12.0$ & 14.5 & $1.8[1.8,4.6]$ & $63.5[45.0,64.0]$ & $25.2 \pm 25.2$ & 24.0 & $2.3[2.3,8.9]$ & $\begin{array}{c}117 \\
{[111,117]}\end{array}$ \\
\hline \multicolumn{13}{|l|}{ Progesterone metabolites } \\
\hline Pregnenolone & $14.2 \pm 10.0$ & 12.5 & $2.8[2.6,4.2]$ & $53.0[41.3,54]$ & $18.0 \pm 14.7$ & 14.9 & $1.9[1.9,3.3]$ & $65.3[57.3,65.5]$ & $27.9 \pm 42.4$ & 16.9 & $4.2[4.2,6.2]$ & $\begin{array}{c}265 \\
{[94.2,265]}\end{array}$ \\
\hline 17-Hydroxypregnanolone & $91.0 \pm 76.4$ & 79.5 & $14.6[9.1,23.1]$ & $229[202,599]$ & $88.3 \pm 55.5$ & 72.8 & $18.8[18.7,41.9]$ & $247[238,247]$ & $189 \pm 253$ & 113 & $34.1[34.2,50.6]$ & $\begin{array}{c}1298 \\
1051,1298]\end{array}$ \\
\hline Pregnanediol & $1269 \pm 830$ & 1110 & $215[154,416]$ & $4257[3093,4405]$ & $1765 \pm 1291$ & 1461 & $459[457,633]$ & $7514[4181,7602]$ & $3982 \pm 3762$ & 2877 & $785[779,1162]$ & [11216, 21112] \\
\hline Pregnanetriol & $209 \pm 114$ & 191 & $53.9[34.2,80.9]$ & $455[378,774]$ & $182 \pm 94.0$ & 153 & $35.8[34.6,99.6]$ & $480[443,481]$ & $287 \pm 321$ & 191 & $53.9[53.4,89.2]$ & $\begin{array}{c}1865 \\
{[970,1865]}\end{array}$ \\
\hline 11-Oxopregnanetriol & $2.7 \pm 2.7$ & 2.0 & $0.4[0.3,0.7]$ & $11.8[10.5,14.7]$ & $2.8 \pm 4.4$ & 1.5 & $0.3[0.3,0.7]$ & $26.4[10.0,26.9]$ & $11.3 \pm 29.2$ & 1.9 & $0.2[0.2,0.5]$ & $\begin{array}{c}151 \\
{[99.2,1501]}\end{array}$ \\
\hline
\end{tabular}


Table 4 Steroid hormone metabolite[ $\mu \mathrm{g} / \mathrm{mmol}$ urinary creatinine]

\begin{tabular}{|c|c|c|c|c|c|c|c|c|c|c|c|c|}
\hline \multirow[b]{3}{*}{ move to a separate row } & \multicolumn{4}{|c|}{$1^{\text {st }}$ trimester $(n=81)$} & \multicolumn{4}{|c|}{$2^{\text {nd }}$ trimester $(n=40)$} & \multicolumn{4}{|c|}{$3^{\text {rd }}$ trimester $(n=40)$} \\
\hline & \multirow[b]{2}{*}{$\begin{array}{l}\text { Mean } \pm \\
\text { SD }\end{array}$} & \multirow[b]{2}{*}{ Median } & \multicolumn{2}{|c|}{ Reference intervals } & \multirow[b]{2}{*}{$\begin{array}{l}\text { Mean } \pm \\
\text { SD }\end{array}$} & \multirow[b]{2}{*}{ Median } & \multicolumn{2}{|c|}{ Reference intervals } & \multirow[b]{2}{*}{ Mean \pm SD } & \multirow[b]{2}{*}{ Median } & \multicolumn{2}{|c|}{ Reference intervals } \\
\hline & & & $\begin{array}{l}2.5^{\text {th }} \text { percentile } \\
{[90 \% \mathrm{Cl}]}\end{array}$ & $\begin{array}{l}97.5^{\text {th }} \text { percentile } \\
{[90 \% \mathrm{Cl}]}\end{array}$ & & & $\begin{array}{l}2.5^{\text {th }} \text { percentile } \\
{[90 \% \mathrm{Cl}]}\end{array}$ & $\begin{array}{l}97.5^{\text {th }} \text { percentile } \\
{[90 \% \mathrm{Cl}]}\end{array}$ & & & $\begin{array}{l}2.5^{\text {th }} \text { percentile } \\
{[90 \% \mathrm{Cl}]}\end{array}$ & $\begin{array}{l}97.5^{\text {th }} \text { percentile } \\
{[90 \% \mathrm{Cl}]}\end{array}$ \\
\hline $\begin{array}{l}\text { Tetrahydro-11- } \\
\text { deoxycortisol }\end{array}$ & $17.1 \pm 10.1$ & 15.5 & $2.7[1.2,6.9]$ & $43.1[35.1,62.5]$ & $23.2 \pm 11.5$ & 21.2 & $5.2[5.1,11.3]$ & $56.2[51.9,56.3]$ & $37.1 \pm 27.4$ & 31.6 & $8.9[8.9,11.4]$ & $146[110,147]$ \\
\hline $\begin{array}{l}\text { Tetrahydro-11- } \\
\text { deoxycorticosterone }\end{array}$ & $12.5 \pm 8.8$ & 10.2 & $1.7[0.9,3.8]$ & $40.1[33.9,46.6]$ & $35.3 \pm 102$ & 16.1 & $4.1[4.1,5.9]$ & $643[466,629]$ & $588 \pm 1681$ & 31.6 & $6.1[6.1,14.7]$ & $8789[5075,8875]$ \\
\hline \multicolumn{13}{|l|}{ Corticosterone metabolites } \\
\hline Tetrahydroaldosterone & $15.4 \pm 10.7$ & 12.0 & $2.7[2.1,5.0]$ & $49.8[36.5,65.6]$ & $18.2 \pm 19.5$ & 11.4 & $1.6[1.6,3.9]$ & $114[40.6,116]$ & $22.9 \pm 16.6$ & 18.2 & $2.1[2.1,7.1]$ & $87.1[55.1,87.9]$ \\
\hline $\begin{array}{l}\text { Tetrahydro-11- } \\
\text { dehydrocorticosterone }\end{array}$ & $33.6 \pm 23.7$ & 26.7 & $3.9[3.2,10.9]$ & $108[88.5,130]$ & $35.1 \pm 19.3$ & 30.3 & $10.2[10.1,15.8]$ & $89.4[87.1,89.4]$ & $88.0 \pm 88.9$ & 56.1 & $13.4[13.3,27.4]$ & $385[371,386]$ \\
\hline Tetrahydrocorticosterone & $23.1 \pm 12.7$ & 21.4 & $3.4[3.0,8.1]$ & $57.8[48.5,63.4]$ & $25.8 \pm 13.2$ & 22.3 & $3.3[3.2,8.5]$ & $57.9[55.0,58.0]$ & $83.0 \pm 107.3$ & 43.4 & $15.2[15.1,22.5]$ & $586[344,591]$ \\
\hline $\begin{array}{l}5 \text { a-Tetrahydro- } \\
\text { corticosterone }\end{array}$ & $51.5 \pm 39.3$ & 39.8 & $5.9[4.3,13.9]$ & $189[143,194]$ & $33.8 \pm 21.6$ & 30.0 & $1.7[1.6,9.8]$ & $93.0[91.7,93]$ & $59.0 \pm 62.4$ & 33.8 & $5.6[5.6,15.0]$ & $304[241,306]$ \\
\hline
\end{tabular}


Table 5 Steroid hormone metabolite[ $\mu \mathrm{g} / \mathrm{mmol}$ urinary creatinine]

\begin{tabular}{|c|c|c|c|c|c|c|c|c|c|c|c|c|}
\hline \multirow[b]{3}{*}{ move to a separate row } & \multicolumn{4}{|c|}{$1^{\text {st }}$ trimester $(n=81)$} & \multicolumn{4}{|c|}{$2^{\text {nd }}$ trimester $(n=40)$} & \multicolumn{4}{|c|}{$3^{\text {rd }}$ trimester $(n=40)$} \\
\hline & \multirow[b]{2}{*}{$\begin{array}{l}\text { Mean } \pm \\
\text { SD }\end{array}$} & \multirow[b]{2}{*}{ Median } & \multicolumn{2}{|c|}{ Reference intervals } & \multirow[b]{2}{*}{$\begin{array}{l}\text { Mean } \pm \\
\text { SD }\end{array}$} & \multirow[b]{2}{*}{ Median } & \multicolumn{2}{|c|}{ Reference intervals } & \multirow[b]{2}{*}{$\begin{array}{l}\text { Mean } \pm \\
\text { SD }\end{array}$} & \multirow[b]{2}{*}{ Median } & \multicolumn{2}{|c|}{ Reference intervals } \\
\hline & & & $\begin{array}{l}2.5^{\text {th }} \text { percentile } \\
{[90 \% \mathrm{Cl}]}\end{array}$ & $\begin{array}{l}97.5^{\text {th }} \text { percentile } \\
{[90 \% \mathrm{Cl}]}\end{array}$ & & & $\begin{array}{l}2.5^{\text {th }} \text { percentile } \\
{[90 \% \mathrm{Cl}]}\end{array}$ & $\begin{array}{l}97.5^{\text {th }} \text { percentile } \\
{[90 \% \mathrm{Cl}]}\end{array}$ & & & $\begin{array}{l}2.5^{\text {th }} \text { percentile } \\
{[90 \% \mathrm{Cl}]}\end{array}$ & $\begin{array}{l}97.5^{\text {th }} \text { percentile } \\
{[90 \% \mathrm{Cl}]}\end{array}$ \\
\hline Cortisone & $34.6 \pm 25.2$ & 28.4 & $4.7[4.4,10.2]$ & $110[97.7,113]$ & $64.5 \pm 39.8$ & 53.3 & $14.1[14.0,25.8]$ & $165[161,165]$ & $79.1 \pm 43.0$ & 82.3 & $4.8[4.7,22.6]$ & $180[144,181]$ \\
\hline Tetrahydrocortisone & $368 \pm 165$ & 349 & $77.6[73.4,162]$ & $780[644,624]$ & $308 \pm 190$ & 255 & $73.8[73.4,90.5]$ & $948[926,947]$ & $321 \pm 210$ & 267 & $5.9[4.7,101]$ & $923[748,922]$ \\
\hline a-Cortolon & $227 \pm 170$ & 211 & $27.6[17.7,35.8]$ & $715[641,725]$ & $112 \pm 138$ & 75.9 & $15.7[15.7,26.0]$ & $826[583,825]$ & $148 \pm 196$ & 68.7 & $17.5[17.5,22.5]$ & $752[741,752]$ \\
\hline$\beta$-Cortolon & $47.9 \pm 37.9$ & 43.4 & $2.5[1.5,5.1]$ & $141[122,167]$ & $27.5 \pm 36.5$ & 13.3 & $3.8[3.8,4.8]$ & $179[148,178]$ & $35.0 \pm 55.1$ & 11.2 & $3.6[3.5,7.5]$ & $303[122,308]$ \\
\hline Cortisone & $34.6 \pm 25.2$ & 28.4 & $4.7[4.4,10.2]$ & $110[97.7,113]$ & $64.5 \pm 39.8$ & 53.3 & $14.1[14.0,25.8]$ & $165[161,165]$ & $79.1 \pm 43.0$ & 82.3 & $4.8[4.7,22.6]$ & $180[144,181]$ \\
\hline 20a-Dihydrocortisone & $5.4 \pm 3.2$ & 4.3 & $1.5[1.4,2.2]$ & $14.9[12.9,15.2]$ & $8.2 \pm 3.8$ & 7.2 & $2.4[2.4,4.2]$ & $15.0[14.3,15.0]$ & $9.8 \pm 4.7$ & 9.4 & $1.4[1.4,4.3]$ & $19.1[18.9,19.1]$ \\
\hline 20ß-Dihydrocortisone & $22.2 \pm 11.9$ & 21.2 & $4.2[3.7,8.2]$ & $52.7[49.9,54.2]$ & $34.0 \pm 16.5$ & 35.3 & $5.7[5.6,12.6]$ & $70.0[67.4,70.0]$ & $45.8 \pm 26.9$ & 37.7 & $1.8[1.7,14.2]$ & $109[104,109]$ \\
\hline \multicolumn{13}{|l|}{ Cortisol metabolites } \\
\hline Cortisol & $29.1 \pm 23.4$ & 20 & $4.7[1.5,7.6]$ & $102[83.7,119]$ & $52.3 \pm 42.3$ & 38.8 & $7.9[7.8,17.3]$ & $210[168,210]$ & $98.2 \pm 109$ & 69.6 & $2.7[2.5,24.5]$ & $641[285,650]$ \\
\hline Tetrahydrocortisol & $191 \pm 99.6$ & 178 & $26.2[22.2,73.1]$ & $405[389,519]$ & $136 \pm 68.1$ & 131 & $31.4[31.2,58.8]$ & $296[277,296]$ & $182 \pm 202$ & 123 & $15.7[15.6,52.9]$ & $923[823,926]$ \\
\hline 5a-Tetrahydrocortisol & $126 \pm 111$ & 90.0 & $13.2[9.6,27.8]$ & $412[379,538]$ & $54.7 \pm 42.2$ & 54.7 & $4.6[4.6,18.4]$ & $158[157,158]$ & $46.7 \pm 55.2$ & 29.2 & $1.3[1.2,10.3]$ & $258[218,259]$ \\
\hline a-Cortol & $55.5 \pm 32.0$ & 49.9 & $8.9[8.2,18.5]$ & $145[111,192]$ & $37.4 \pm 17.9$ & 34.6 & $7.1[6.9,18.6]$ & $107[73.5,108]$ & $51.1 \pm 56.5$ & 35.1 & $6.0[5.9,12]$ & $248[235,248]$ \\
\hline$\beta$-Cortol & $45.6 \pm 22.8$ & 39.9 & $14.9[11.8,21.0]$ & $118[10.0,122]$ & $32.4 \pm 16.7$ & 26.6 & $6.9[6.7,15.2]$ & $80.0[68.2,80.3]$ & $36.3 \pm 33.5$ & 24.1 & $6.3[6.2,13.2]$ & $187[101,189]$ \\
\hline 20a-Dihydrocortisol & $30.0 \pm 33.7$ & 19.7 & $1.9[1.8,6.6]$ & $180[117,215]$ & $60.4 \pm 60.2$ & 37.3 & $1.7[1.6,9.5]$ & $258[246,258]$ & $145 \pm 203$ & 74.8 & $5.1[4.9,18.3]$ & $1049[623,1060]$ \\
\hline
\end{tabular}


Table 6 Postpartum urinary steroid hormone concentrations

\begin{tabular}{|c|c|c|c|c|}
\hline \multirow{3}{*}{$\begin{array}{l}\text { Steroid } \mu \mathrm{g} \text { per mmol creatinine } \\
\text { move to a separate row }\end{array}$} & \multicolumn{4}{|c|}{6 weeks postpartum $(n=40)$} \\
\hline & \multirow[b]{2}{*}{ Mean \pm SD } & \multirow[b]{2}{*}{ Median } & \multicolumn{2}{|l|}{ Reference intervals } \\
\hline & & & $2.5^{\text {th }}$ percentile $[90 \% \mathrm{Cl}]$ & $97.5^{\text {th }}$ percentile $[90 \% \mathrm{Cl}]$ \\
\hline Androsterone & $130 \pm 116$ & 99.8 & $6.5[6.2,24.2]$ & $524[482,525]$ \\
\hline Etiocholanolone & $180 \pm 120$ & 149 & $19.4[19.3,42.7]$ & $456[423,457]$ \\
\hline 11-Oxo-Etiocholanolone & $51.0 \pm 35.1$ & 42.1 & $8.0[8.0,14.9]$ & $156[149,156]$ \\
\hline $11 \beta$-Hydroxy-Androsterone & $77.5 \pm 51.4$ & 71.1 & $4.4[4.2,19.1]$ & $262[149,156]$ \\
\hline 11ß-Hydroxy-Etiocholanolone & $46.4 \pm 36.6$ & 38.3 & $4.8[4.8,9.3]$ & $161[150,161]$ \\
\hline Dehydroepiandrosterone & $33.5 \pm 57.1$ & 10.4 & $0.8[0.8,2.7]$ & $291[160,294]$ \\
\hline 5-Androstene-3 $\beta, 17 \beta$-diol & $14.2 \pm 15.1$ & 9.4 & $0.4[0.4,1.6]$ & $68.0[50.0,68.4]$ \\
\hline 5-Androstene-3 $\beta, 17 \beta$-triol & $9.8 \pm 21.5$ & 3.1 & $0.2[0.2,0.6]$ & $113[76.5,114]$ \\
\hline 16a-Hydroxy-dehydroepiandrostrerone & $46.8 \pm 86.8$ & 22.8 & $0.9[0.9,3.1]$ & $502[212,510]$ \\
\hline 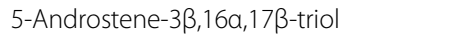 & $40.4 \pm 45.9$ & 29.6 & $3.7[3.6,9.5]$ & $271[116,275]$ \\
\hline 5-Pregnene-3 $\beta, 16 a, 17,20 a$-triol & $13.5 \pm 12.5$ & 10.7 & $0.4[0.4,1.3]$ & $59.5[38.9,60.0]$ \\
\hline Testosterone & $3.7 \pm 5.7$ & 1.4 & $0.1[0.1,0.5]$ & $26.0[22.9,26.1]$ \\
\hline 5a-Dihydrotestosterone & $2.5 \pm 2.3$ & 2.2 & $0.1[0.1,0.6]$ & $12.4[8.2,12.3]$ \\
\hline \multicolumn{5}{|l|}{ Oestrogen metabolites } \\
\hline Estriol & $119 \pm 339$ & 17.6 & $0.9[0.9,1.4]$ & $2011[557,2049]$ \\
\hline 17ß-Estradiol & $2.9 \pm 9.6$ & 0.5 & $0.1[0.05,0.1]$ & $58.7[9.0,60.0]$ \\
\hline \multicolumn{5}{|l|}{ Progesterone precursor and metabolites } \\
\hline Pregnenolone & $9.8 \pm 13.4$ & 4.7 & $0.2[0.2,0.7]$ & $52.5[49.7,52.2]$ \\
\hline 17-Hydroxypregnanolone & $20.2 \pm 41.4$ & 9.9 & $0.9[0.9,2.4]$ & $257[76.6,262]$ \\
\hline Pregnanediol & $257 \pm 668$ & 76.2 & $10.4[10.3,29.5]$ & $4052[1173,4126]$ \\
\hline Pregnanetriol & $56.0 \pm 49.7$ & 44.3 & $5.9[5.7,20.8]$ & $297[144,301]$ \\
\hline 11-Oxo-Pregnanetriol & $2.8 \pm 3.4$ & 1.6 & $0.4[0.4,0.7]$ & $17.7[10.1,17.8]$ \\
\hline \multicolumn{5}{|l|}{ 11-Deoxysteroid metabolites } \\
\hline Tetrahydro-11-deoxycortisol & $11.1 \pm 8.6$ & 9.8 & $0.7[0.7,3.0]$ & $43.1[30.6,42.8]$ \\
\hline Tetrahydro-11-deoxycorticosterone & $28.2 \pm 160$ & 1.3 & $0.1[0.1,0.4]$ & $989[27.0,1014]$ \\
\hline \multicolumn{5}{|l|}{ Corticosterone metabolites } \\
\hline Tetrahydroaldosterone & $4.2 \pm 4.6$ & 2.6 & $0.5[0.5,1.1]$ & $21.9[19.3,21.9]$ \\
\hline Tetrahydro-11-dehydrocorticosterone & $20.7 \pm 23.5$ & 14.6 & $0.4[0.4,3.3]$ & $139[53.9,142]$ \\
\hline Tetrahydrocorticosterone & $23.8 \pm 16.7$ & 20.1 & $2.2[2.2,6.8]$ & $71.3[69.3,71.3]$ \\
\hline 5a-Tetrahydrocorticosterone & $35.7 \pm 28.1$ & 28.8 & $1.7[1.6,6.3]$ & $143[113,143]$ \\
\hline \multicolumn{5}{|l|}{ Cortisone metabolite } \\
\hline Cortisone & $28.2 \pm 20.1$ & 23.4 & $1.0[1.0,10.3]$ & $101[74.8,101]$ \\
\hline Tetrahydrocortisone & $397 \pm 233$ & 373 & $58.8[58.2,120]$ & $1034[906,1037]$ \\
\hline a-Cortolon & $122 \pm 117$ & 90.5 & $18.1[18,33.5]$ & $707[286,718]$ \\
\hline$\beta$-Cortolon & $30.6 \pm 40.4$ & 6.1 & $0.2[0.2,1.5]$ & $156[141,155]$ \\
\hline 20a-Dihydrocortisone & $3.4 \pm 2.7$ & 2.7 & $0.2[0.2,1.1]$ & $13.5[9.7,13.6]$ \\
\hline 20ß-Dihydrocortisone & $9.2 \pm 6.9$ & 8.1 & 0.5 [0.4. 3.1] & $34.3[24.5,34.5]$ \\
\hline \multicolumn{5}{|l|}{ Cortisol metabolites } \\
\hline Cortisol & $20.8 \pm 19.1$ & 13.8 & $0.9[0.8,4.1]$ & $99.7[66.7,99.7]$ \\
\hline Tetrahydrocortisol & $256 \pm 153$ & 243 & $30.7[30.3,80.3]$ & $723[599,726]$ \\
\hline 5a-Tetrahydrocortisol & $97.1 \pm 81.2$ & 78.3 & $5.8[5.4,22.7]$ & $381[302,383]$ \\
\hline a-Cortol & $41.1 \pm 32.6$ & 35.5 & $3.9[3.8,17.7]$ & $203[98.2,206]$ \\
\hline
\end{tabular}


Table 6 Postpartum urinary steroid hormone concentrations (Continued)

\begin{tabular}{lllcr}
\hline$\beta$-Cortol & $55.3 \pm 29.2$ & 50.1 & $10.0[9.9,25.8]$ & $130[114,130]$ \\
20a-Dihydrocortisol & $17.3 \pm 22.6$ & 9.3 & $0.2[0.2,3]$ & $127[50.7,127]$ \\
63-Hydroxycortisol & $149 \pm 25.8$ & 26.5 & $0.9[0.9,8.1]$ & $149[77.9,149]$ \\
18-Hydroxycortisol & $322 \pm 55.1$ & 41.7 & $1.5[1.4,4.9]$ & $322[130,322]$ \\
\hline
\end{tabular}

and chromatography. The percentage recovery following extraction for all the assays was $>90 \%$. The samples were derivatised to form methyloxime-trimethylsilyl ethers. The derivatives were purified by gel filtration on Lipidex 5000 (Perkin Elmer, USA) columns. Analysis was performed on a Hewlett-Packard gas chromatograph 6890 (HewlettPackard, Palo Alto, California, USA) with mass selective detector 5973 by selective ion monitoring (SIM). A steroid mixture containing a known amount of all steroid metabolites to be measured was analysed on a regular basis to act as a calibration standard. To control extraction variability, a control sample was run on each extraction panel. All steroid concentrations were normalised to urine creatinine values.

\section{Assessment of urinary creatinine concentrations}

Urinary creatinine concentrations were measured either in the clinical laboratory or by a validated QuantiChromTM Creatinine Assay Kit (DICT-500; BioAssay Systems, Switzerland), with intra-assay coefficient of variations for both assays $<5 \%$. Both assays were based on the same method and reference samples were analysed on both platforms to ensure similar results; measurement variability between the 2 techniques was less than $5 \%$.

\section{Statistics}

Descriptive statistics, including upper and lower bounds of reference intervals were calculated for each metabolite separately by trimester and postpartum using SPSS version 22. Reference intervals are described as the 2.5 and 97.5th percentiles, centred around the median with their corresponding $90 \%$ confidence intervals [17]. Virtanen et al., states that sample sixes above 30 had smaller changes in SDs and CVs in reference intervals and thus at least 40 samples for each time point were used [14]. Data was found to be non-parametric following the Kolmogorov-Smirnov test for normality of data distribution, thus robust methods, with bootstrapping was used, which utilises an interactive process to estimate location and spread of the data [18].

\section{Results}

\section{Participants}

Basic demographic, obstetric and pregnancy description data of the women in this study are detailed in Table 1. Overall, the 3 cohorts were well matched with no significant differences observed for all demographic data. The majority of women who participated in this study were White European (>95 \%) from all 3 centres.

\section{Reference intervals}

All steroid hormone concentrations, normalised to creatinine, grouped by trimester and steroid hormone are given in Tables 2, 3, 4 and 5 and the postpartum values are presented in Table 6, including the 2.5 and $97.5 \%$ reference intervals. For ease of clinical use, the mean and median value for each steroid hormone metabolite has also been calculated, normalised to creatinine concentrations (Tables 2, 3, 4, 5 and 6).

\section{Androgen metabolites}

For clarity, androgens are presented in 2 graphs as follows: Fig. 1a: androsterone, etiocholanolone, 11-oxoetiocholanolone 11 $\beta$-hydroxy-androsterone, dehydroepiandrosterone, $16 \alpha$-hydroxydehydroepiandrosterone and 5 -androstene- $3 \beta, 16 \alpha, 17 \beta$-triol. Figure 1b: $11 \beta$-hydroxy-etiocholanolone, 5-androstene-3 $\beta, 16 \alpha, 17 \beta$-diol, 5androstene-3 $\beta, 16 \alpha, 17 \beta$-triol, 5 -pregnene-3 $\beta, 16 \alpha, 17,20 \alpha$ triol, testosterone and $5 \alpha$-dihydrotestosterone.

\section{Oestrogen metabolites}

Both estriol and $17 \beta$-estradiol differed between the trimesters and postpartum (Fig. 2a); with the highest concentrations in the third trimester, followed by a dramatic decrease postpartum, lower than first trimester concentrations (Fig. 1a).

\section{Progesterone precursor and metabolites}

The progesterone percursor, pregnenolone and all the progesterone metabolites (17-hydroxypregnanolone, pregnanetriol, pregnanediol, and 11-oxo-pregnanetriol) are presented in Fig. 2b. All metabolites increased between the $2^{\text {nd }}$ and $3^{\text {rd }}$ trimester, then subsequently fell postpartum.

\section{1-deoxysteroid hormone metabolites}

Both tetrahydro-11-deoxycortisol and tetrahydro-11deoxycorticosterone concentrations were increased with gestational age and decreased postpartum (Fig. 3a). 

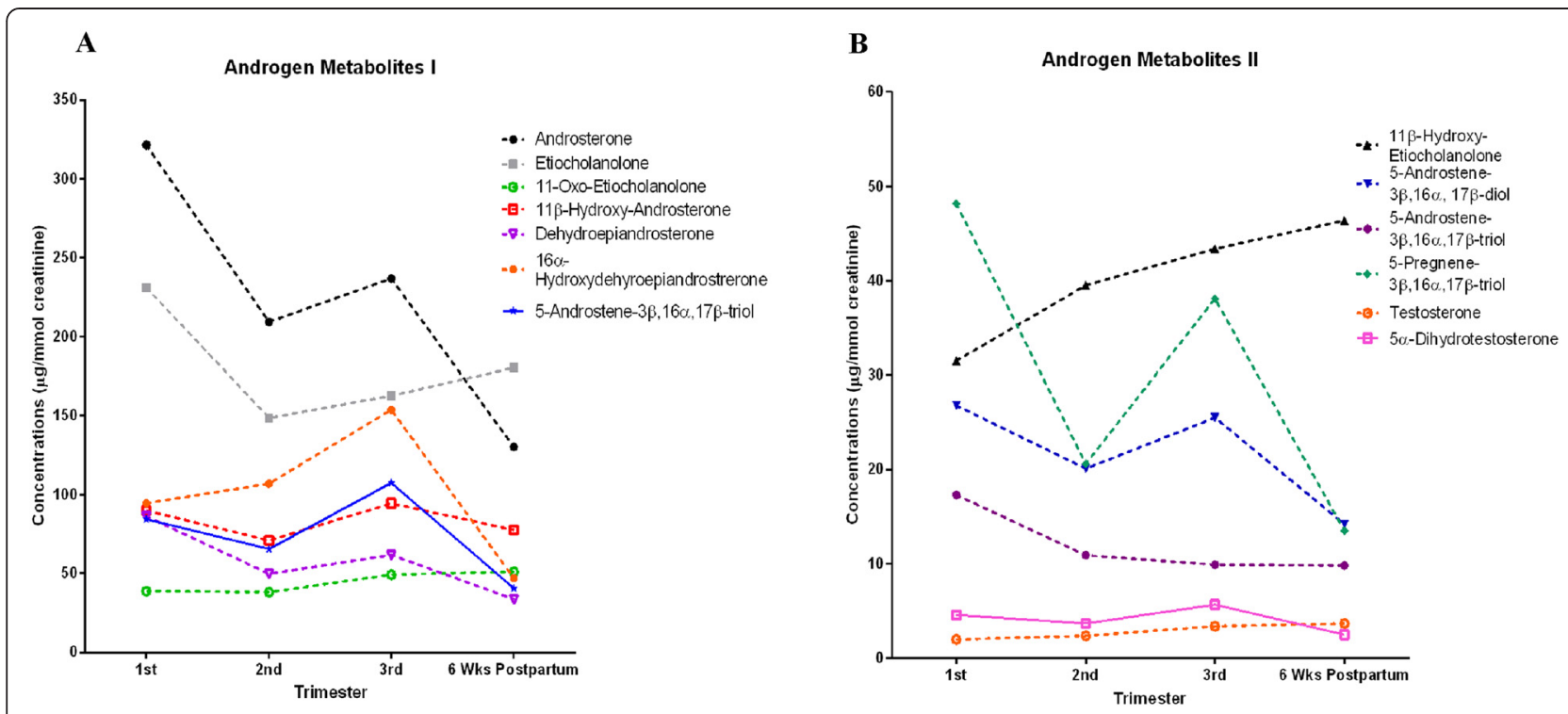

Fig. $1 \mathbf{a}$ and $\mathbf{b}$ Urinary concentrations of androgen metabolites in first, second, third trimester and six weeks postpartum. Each point is presented as mean corrected for creatinine concentrations ( $\mathrm{g} / \mathrm{mmol}$ creatinine)

\section{Corticosterone metabolites}

All metabolites differed between trimesters (Fig. 3b). Tetrahydroaldosterone and tetrahydrocorticosterone already increased throughout pregnancy and then subsequently decreased 6-weeks postpartum (Fig. 3b).

\section{Cortisol and cortisone metabolites}

For clarity, cortisol and cortisone metabolites are presented in 2 graphs as follows: Fig. 4a: cortisol, tetrahydrocortisol, $5 \alpha$-tetrahydrocortisol, $\alpha$-cortol, $\beta$-cortol, $20 \alpha$-dihydrocortisol, $6 \beta$-hydroxycortisol and 18-hydroxycortisol. Figure 4b: cortisone, tetrahydrocortisone, $\alpha$-cortolon, $\beta$-cortolon, $20 \alpha-$ dihydrocortisone and 20ß-dihydrocortisone. The most dramatic change was the increase in 18-hydroxycortisol during the 3 trimesters and postpartum.

For all steroid hormone metabolites measured, we tested whether any fetal gender specific differences were detectable. No significant differences $(P<0.05)$ were observed for any steroid hormone metabolites.

\section{Discussion}

We have investigated the changes in spot urinary concentrations of the main steroid hormone metabolites throughout normal pregnancy and 6 weeks postpartum

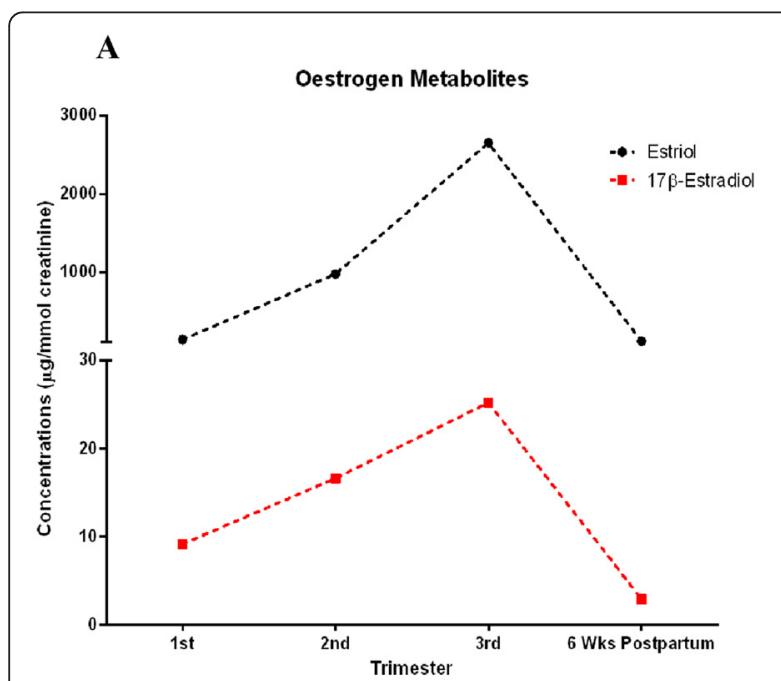

$\mathbf{B}$

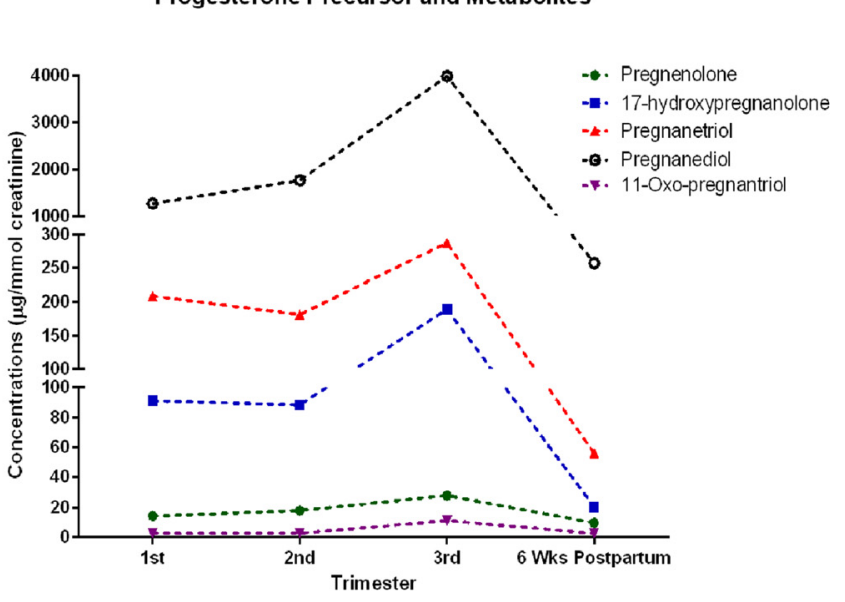

Fig. 2 Urinary concentrations of (a) oestrogen metabolites and (b) progesterone precursor (pregnenolone) and progesterone metabolites in first, second, third trimester and six weeks postpartum. Each point is presented as mean corrected for creatinine concentrations ( $\mu \mathrm{g} / \mathrm{mmol} \mathrm{creatinine})$ 


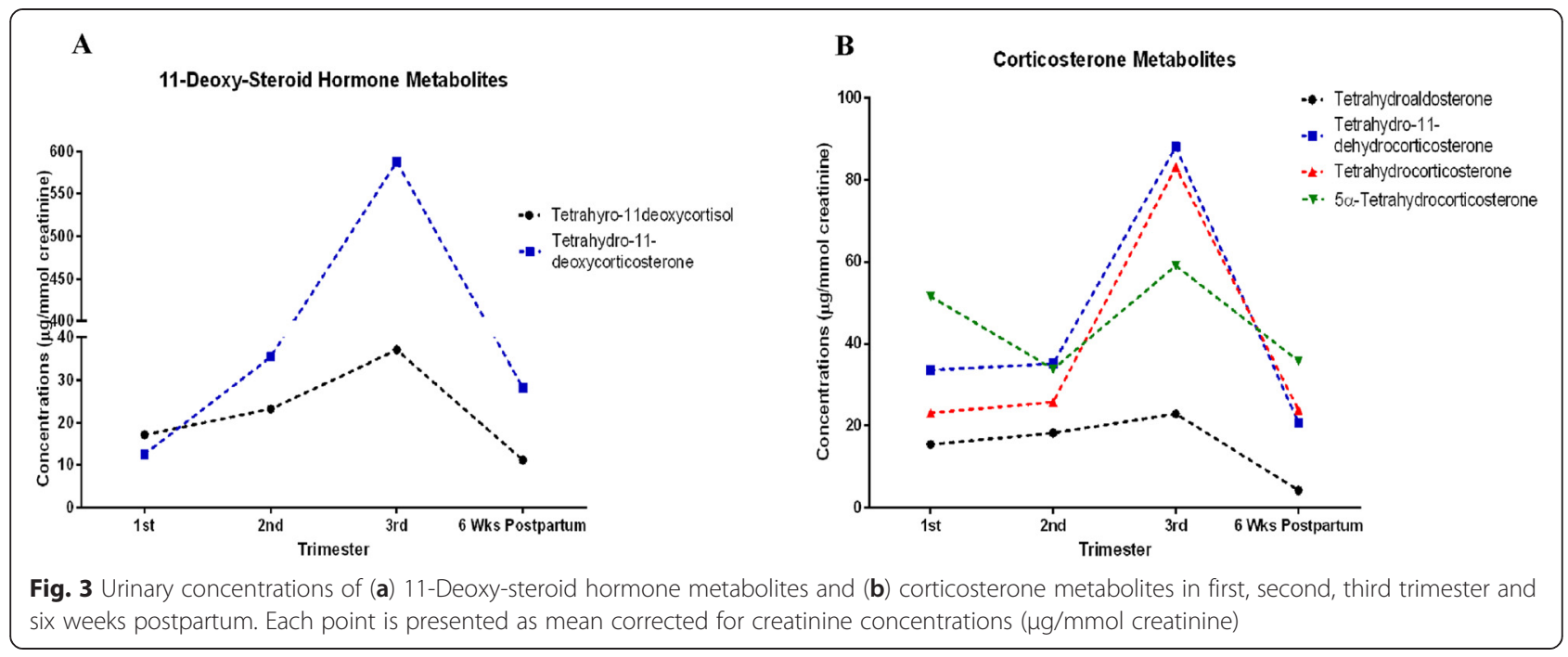

using a validated GC-MS method. Furthermore, we have used appropriate analysis techniques to calculate clinically relevant gestation-specific reference intervals. These data illustrate the profound changes in maternal steroidogenesis during pregnancy and indicate normal functional changes such as of the hypothalamic-pituitarygonadal axis during pregnancy. A relationship between pregnancy outcome and steroid hormone availability such as for aldosterone has been established in the past by our group for aldosterone and cortisol [19, 20]. It have been known for many years that during the first 9 weeks of pregnancy, the corpus luteum and, the maternal ovary and adrenal cortex contribute to circulating concentrations of maternal hormones [21, 22]. After this time, the placenta becomes the predominant source of many maternal steroids [23].

These can then be used to improve management of steroid hormone related diseases in pregnancy, as well as encourage the appreciation of the changes that occur in steroid hormones during pregnancy. It will also allow clinicians to use gestation-specific reference ranges for management. Several steroid hormone metabolites are often combined to provide the clinician with a general picture of the individual's steroid hormone production. This is useful as first point of investigation to obtain an integrated picture of the patient, to aid the identification of inherited metabolic disorders of steroid metabolism, and to perform non-invasive diagnostics. If normal values for pregnancy are established, even prenatal diagnosis of fetal disease might be feasible. Given the altered steroid hormone profile in pregnancy, these normal values might also allow discrimination between acquired, such as adrenal adenomas, and inborn errors of metabolism [7] (detailed on Table 7). Furthermore, some metabolites are still the subject of research investigations in the future. This could contribute to our knowledge of developmental
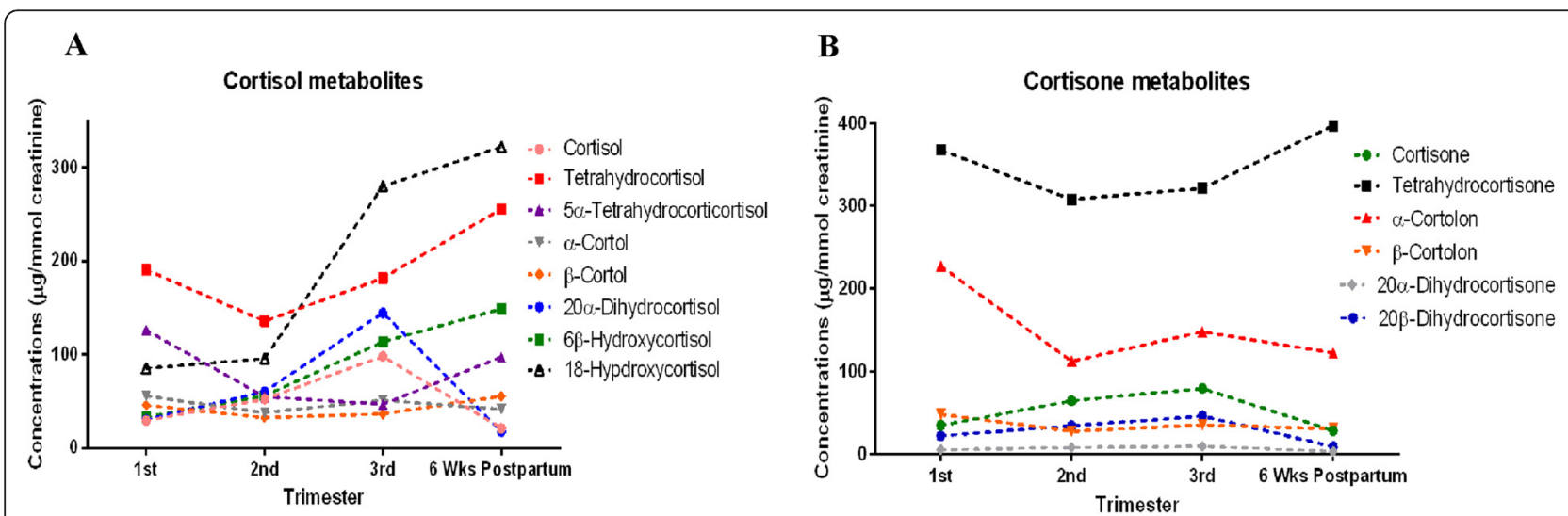

Fig. 4 Urinary concentrations (a) cortisol metabolites and (b) cortisone metabolites in first, second, third trimester and six weeks postpartum. Each point is presented as mean corrected for creatinine concentrations $(\mu \mathrm{g} / \mathrm{mmol}$ creatinine) 
Table 7 Assignment of steroid hormone metabolites to their main reference

\begin{tabular}{|c|c|c|}
\hline Full trivial name & Reference & Analyzed enzyme system \\
\hline \multicolumn{3}{|l|}{ Androgen metabolites } \\
\hline Androsterone & $\begin{array}{l}\text { Androstenedione, testosterone, } \\
5 \text { a-dihydrotestosterone, dehydroepiandrosterone }\end{array}$ & CYP17A1, POR, SRD5A2, HSD17B3 \\
\hline Etiocholanolone & Testosterone, dehydroepiandrosterone & CYP17A1, POR, SRD5A2, HSD17B3 \\
\hline $11 \beta$-Hydroxy-androsterone & $11 \beta$-hydroxy-androstenedione, cortisol & \\
\hline Dehydroepiandrosterone & Dehydroepiandrosterone & HSD3B2 \\
\hline 5-Androstene-3 $\beta, 17 \beta$-diol & Testosterone, dehydroepiandrosterone & \\
\hline 5-Androstene-3 $\beta, 17 \beta$-triol & Testosterone, dehydroepiandrosterone & \\
\hline 16a-Hydroxy-dehydroepiandrosterone & Dehydroepiandrosterone & \\
\hline 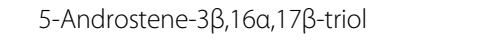 & Testosterone, dehydroepiandrosterone & \\
\hline 11ß-Hydroxy-etiocholanolone & Testosterone, dehydroepiandrosterone, cortisol & \\
\hline 11-Oxo-etiocholanolone & Testosterone, dehydroepiandrosterone, cortisol & \\
\hline Testosterone & Testosterone & \\
\hline 5a-Dihydrotestosterone & Testosterone & \\
\hline \multicolumn{3}{|l|}{ Oestrogen metabolites } \\
\hline Estriol & Estradiol & \\
\hline $17 \beta$-Estradiol & Estradiol & \\
\hline \multicolumn{3}{|l|}{ Progesterone metabolites } \\
\hline Pregnenolone & Pregnenolone & \\
\hline 17-Hydroxypregnanolone & 17-Hydroxy-progesterone & CYP17A1, POR \\
\hline Pregnanediol & Pregnenolone & POR \\
\hline Pregnanetriol & 17-Hydroxy-progesterone & CYP21A2, POR \\
\hline 11-Oxopregnanetriol & 17-Hydroxy-progesterone & CYP21A2 \\
\hline 5-Pregnene-3 $\beta, 16 a, 17,20 a$-triol & 17-Hydroxy-pregnenolone & HSD3B2 \\
\hline \multicolumn{3}{|l|}{ 11-Deoxysteroid hormone metabolites } \\
\hline Tetrahydro-11-deoxycortisol & 11-Deoxycortisol & CYP11B1 \\
\hline Tetrahydro-11-deoxycorticosterone & Corticosterone & \\
\hline \multicolumn{3}{|l|}{ Corticosterone metabolites } \\
\hline Tetrahydrocorticosterone & 11-Deoxycorticosterone & CYP17A1, POR, SRD5A2 \\
\hline 5a-Tetrahydrocorticosterone & 11-Deoxycorticosterone & CYP17A1, POR, SRD5A2 \\
\hline Tetrahydro-11-dehydrocorticosterone & Corticosterone & CYP17A1, POR \\
\hline Tetrahydroaldosterone & Aldosterone & \\
\hline \multicolumn{3}{|l|}{ Cortisol metabolites } \\
\hline Cortisol & Cortisol & HSD11B2 \\
\hline Tetrahydrocortisol & Cortisol & $\begin{array}{l}\text { CYP21A2, CYP11B1, CYP17A1, HSD3B2, POR, } \\
\text { HSD11B2, H6PDH, HSD17B3 }\end{array}$ \\
\hline 5a-Tetrahydrocortisol & Cortisol & $\begin{array}{l}\text { CYP21A2, CYP11B1, CYP17A1, HSD3B2, POR, } \\
\text { HSD11B2, H6PDH, HSD17B3, SRD5A2 }\end{array}$ \\
\hline a-Cortol & Cortisol & HSD11B2 \\
\hline$\beta$-Cortol & Cortisol & HSD11B2 \\
\hline 20a-Dihydrocortisol & Cortisol & \\
\hline $6 \beta$-Hydroxycortisol & Cortisol & \\
\hline 18-Hydroxycortisol & Cortisol & \\
\hline
\end{tabular}


Table 7 Assignment of steroid hormone metabolites to their main reference (Continued)

\begin{tabular}{lll}
\hline $\begin{array}{l}\text { Cortisone metabolites } \\
\text { Cortisone }\end{array}$ & Cortisol, cortisone & HSD11B2 \\
Tetrahydrocortisone & Cortisol, cortisone & CYP21A2, CYP11B1, CYP17A1, HSD3B2, POR, \\
& & HSD11B2, H6PDH, HSD17B3 \\
a-Cortolone & Cortisol, cortisone & HSD11B2 \\
B-Cortolone & Cortisol, cortisone & HSD11B2 \\
20a-Dihydrocortisone & Cortisol, cortisone & \\
20ß-Dihydrocortisone & Cortisol, cortisone & \\
\hline
\end{tabular}

changes in synthesis and metabolism of steroid hormones, such as the early diagnosis of congenital adrenal hyperplasia [24].

The placenta is a site for active steroidogenesis, which depends on highly integrated and active interactions with both mother and fetus. As is well established, progesterone levels are important for normal feto-placental function, playing essential roles in controlling and maintaining the course of normal pregnancy $[25,26]$. The rises in these metabolites during pregnancy and subsequent fall postpartum are thus the normal responses previously described [5]. Equally many of the changes in mineralocorticoids and in glucocorticoids also reflect the normal physiological responses to pregnancy and in preparation for parturition [27].

Androgens derived from the maternal adrenal tissue can undergo aromatization to form oestrogens [28]. Although clinical manifestations of androgen excess are uncommon in pregnant women, any physical changes in these metabolites from the normal changes described should be of concern. After the first 5 to 6 weeks of pregnancy, the major source of estradiol is the placenta, where enzymes convert maternal and fetal dehydroepiandrosterone to estradiol, estrone and estriol [22]. Near term, $50 \%$ of estradiol synthesised in the placenta is derived from precursors in the fetal circulation; the remainder coming from the maternal circulations. This explains the increased levels of this and $17 \beta$-estradiol in the third trimester. It is well known that the expulsion of the placenta causes a decline in steroid levels [23], reflected and confirmed in the postpartum concentrations in the current study.

These normal values reflect known changes in index steroid hormone levels throughout pregnancy (e.g. increases in oestrogen metabolites in the third trimester; Fig. 2a). However, numerous studies might have benefited from the addition of MS-based to conventional RIA- or ELISA-based methods to obtain more accurate levels, by avoiding interference with steroid hormone binding, not missing important metabolites or simply by circumventing cross-reactivity. Furthermore, urinary measurements are not just a momentary picture, but integrate hormone production over a given time span. The high accuracy and specificity will now allow comprehensive clinical and scientific analysis in pregnant women, which has recently been postulated in the endocrine community [29]. The multi-centric setup avoids centre bias, although given the uncomplicated pre-analytics in steroid hormone metabolite analytics; such an issue is quite unlikely. Further advantages of urinary samples include less invasiveness, which is a huge benefit in pediatric and pregnant populations. We also expect that fetal pathologies might be identified by maternal urine sampling in pregnancy. Moreover, blood sampling reflects just a single time point whereas the urine sampling allows a cumulative integrated view of the steroid hormone metabolome over the sampling period. Upon calculation of specific metabolite ratios, conditions of adrenal origin and other disorders might be diagnosed without the need of timed sample collection [7]. A limitation of all steroid studies, including ours, is the high variation between patients, possibly due to the use of spot, as opposed to $24 \mathrm{~h}$ urine collections, and the variable physical activity of participating individuals. Conversely, this is also a strength of the study and obtaining accurate $24 \mathrm{~h}$ urine collections are problematic, particularly during pregnancy and thus spot urine offers a suitable and more practical method for assessment of steroid metabolites. In addition, a large degree of day-to-day variation within individuals has to be considered [30-33]. This study was conducted primarily in a population of white European descent and while the absolute values might be limited in their generality, the hormonal patterns reflecting the HPG and HPA axes might show sufficient similarities even in different genetic backgrounds [31, 33-35]. The addition of samples from 3 different populations, account for variations in diet and salt intakes and thus provides more accurate reference intervals. Future work will establish possible changes in these steroid profiles in $24 \mathrm{~h}$ urine collections and in pathological pregnancies.

\section{Conclusions}

In summary, we have established, as far as we are aware, for the first time, gestation-specific GC-MS based reference values for spot urinary endogenous hormone metabolites 
during pregnancy, which may also be of interest in studies of maternal and fetal health and disease. We have created an easy to use functionally grouped trimester-based table (Tables 2, 3, 4, 5 and 6) for each hormone, which will be of use to those managing and studying women with respect to steroid hormone metabolites in pregnancy.

\section{Competing interests}

The authors declare that they have no competing interest.

\section{Authors' contributions}

$\mathrm{HDM}, \mathrm{NE}, \mathrm{BD}, \mathrm{GC}$, and CG-M performed the research; HDM, and MGM analysed the data. All authors wrote, revised and approved the manuscript.

\section{Acknowledgments}

We thank all the pregnant women that participated in this study. We are grateful for financial support for this study: HDM is supported by an ERA-EDTA Fellowship (ERA LTF 137-2013). GC is supported by an NHS Greater Glasgow \& Clyde Endowment Grant (13REF008). MGM is supported by the Swiss National Foundation (Personal grants: 3200B0-113902/1, 32-135596) The authors also wish to gratefully acknowledge the assistance of staff at all 3 recruiting centres in Bern, Graz and Glasgow for their help with sample collection, which aided successful completion of this project. We also thank Judith Herbst and Marco Groppo for technical help and assistance.

\section{Author details}

'Department of Nephrology, Hypertension, Clinical Pharmacology and Clinical Research, University of Bern, 3010 Berne, Switzerland. ${ }^{2}$ Department of Obstetrics and Gynecology, University Hospital Bern, 3010 Berne, Switzerland. ${ }^{3}$ University of Glasgow, Institute of Cardiovascular and Medical Sciences, Glasgow, UK. ${ }^{4}$ Vivantes Clinic Berlin-Neukölln, Department of Obstetrics, Berlin, Germany. ${ }^{5}$ Division of Hypertension, Department of Nephrology, Hypertension, Clinical Pharmacology and Clinical Research, University of Bern, $\mathrm{CH}-3010$ Berne, Switzerland.

Received: 22 June 2015 Accepted: 27 August 2015

\section{Published online: 04 September 2015}

\section{References}

1. Nussey S, Whitehead S. Endocrinology - an intergrated approach. Oxford: BIOS Scientific Publishers, Ltd; 2001.

2. Buster JE, Abraham GE. The applications of steroid hormone radioimmunoassays to clinical obstetrics. Obstet Gynecol. 1975;46:489-99.

3. Berstein LM. Newborn macrosomy and cancer. Adv Cancer Res. 1988:50:231-78.

4. Gennari-Moser C, Escher G, Kramer S, Dick B, Eisele N, Baumann M, et al. Normotensive blood pressure in pregnancy: the role of salt and aldosterone. Hypertension. 2014;63:362-8.

5. Makieva S, Saunders PT, Norman JE. Androgens in pregnancy: roles in parturition. Hum Reprod Update. 2014;20:542-59.

6. Quattropani C, Vogt B, Odermatt A, Dick B, Frey BM, Frey FJ. Reduced activity of 11 beta-hydroxysteroid dehydrogenase in patients with cholestasis. J Clin Invest. 2001;108:1299-305.

7. Krone N, Hughes BA, Lavery GG, Stewart PM, Arlt W, Shackleton CH. Gas chromatography/mass spectrometry (GC/MS) remains a pre-eminent discovery tool in clinical steroid investigations even in the era of fast liquid chromatography tandem mass spectrometry (LC/MS/MS). J Steroid Biochem Mol Biol. 2010;121:496-504.

8. Hertig A, Liere P, Chabbert-Buffet N, Fort J, Pianos A, Eychenne B, et al. Steroid profiling in preeclamptic women: evidence for aromatase deficiency. Am J Obstet Gynecol. 2010;203:477. e471-479.

9. Fabregat A, Marcos J, Garrostas L, Segura J, Pozo OJ, Ventura R. Evaluation of urinary excretion of androgens conjugated to cysteine in human pregnancy by mass spectrometry. J Steroid Biochem Mol Biol. 2014;139:192-200.

10. Shojaati K, Causevic M, Kadereit B, Dick B, Imobersteg J, Schneider H, et al. Evidence for compromised aldosterone synthase enzyme activity in preeclampsia. Kidney Int. 2004;66:2322-8.
11. Meng LJ, Reyes H, Palma J, Hernandez I, Ribalta J, Sjovall J. Profiles of bile acids and progesterone metabolites in the urine and serum of women with intrahepatic cholestasis of pregnancy. J Hepatol. 1997;27:346-57.

12. Ross MG, Idah R. Correlation of maternal plasma volume and composition with amniotic fluid index in normal human pregnancy. J Matern Fetal Neonatal Med. 2004;15:104-8.

13. Royston P. Constructing time-specific reference ranges. Stat Med. 1991;10:675-90.

14. Virtanen A, Kairisto V, Uusipaikka E. Regression-based reference limits: determination of sufficient sample size. Clin Chem. 1998;44:2353-8.

15. O'Donnell MJ, Yusuf S, Mente A, Gao P, Mann JF, Teo K, et al. Urinary sodium and potassium excretion and risk of cardiovascular events. JAMA. 2011:306:2229-38.

16. Shackleton $\mathrm{CH}$. Mass spectrometry in the diagnosis of steroid-related disorders and in hypertension research. J Steroid Biochem Mol Biol. 1993;45:127-40.

17. Wright EM, Royston P. Calculating reference intervals for laboratory measurements. Stat Methods Med Res. 1999;8:93-112.

18. Horn PS, Pesce AJ. Reference intervals: an update. Clin Chim Acta. 2003;334:5-23.

19. Gennari-Moser C, Khankin EV, Schuller S, Escher G, Frey BM, Portmann CB, et al. Regulation of placental growth by aldosterone and cortisol. Endocrinology. 2011;152:263-71.

20. Aufdenblatten M, Baumann M, Raio L, Dick B, Frey BM, Schneider H, et al. Prematurity is related to high placental cortisol in preeclampsia. Pediatr Res. 2009:65:198-202.

21. Yoshimi T, Strott CA, Marshall JR, Lipsett MB. Corpus luteum function in early pregnancy. J Clin Endocrinol Metab. 1969;29:225-30.

22. Tulchinsky D, Hobel CJ, Yeager E, Marshall JR. Plasma estrone, estradiol, estriol, progesterone, and 17-hydroxyprogesterone in human pregnancy. I. Normal pregnancy. Am J Obstet Gynecol. 1972;112:1095-100.

23. Torrealday S, Taylor HS, Burney RO, Mooney SB, Giudice LC. Endocrinology of pregnancy. (De Groot L, Beck-Peccoz P, Chrousos G eds.), 2015/04/24 edition. South Dartmounth: MDText.com, Inc.; 2000

24. Reisch N, Idkowiak J, Hughes BA, Ivison HE, Abdul-Rahman OA, Hendon LG, et al. Prenatal diagnosis of congenital adrenal hyperplasia caused by P450 oxidoreductase deficiency. J Clin Endocrinol Metab. 2013;98:E528-536.

25. O'Leary P, Boyne P, Flett P, Beilby J, James I. Longitudinal assessment of changes in reproductive hormones during normal pregnancy. Clin Chem. 1991;37:667-72.

26. Tulchinsky D, Korenman SG. The plasma estradiol as an index of fetoplacental function. J Clin Invest. 1971;50:1490-7.

27. Whittle WL, Patel FA, Alfaidy N, Holloway AC, Fraser M, Gyomorey S, et al. Glucocorticoid regulation of human and ovine parturition: the relationship between fetal hypothalamic-pituitary-adrenal axis activation and intrauterine prostaglandin production. Biol Reprod. 2001;64:1019-32.

28. Rainey WE, Rehman KS, Carr BR. Fetal and maternal adrenals in human pregnancy. Obstet Gynecol Clin North Am. 2004;31:817-35.

29. Raff H, Auchus RJ, Findling JW, Nieman LK. Urine free cortisol in the diagnosis of cushing's syndrome: is it worth doing, and if so, how? J Clin Endocrinol Metab. 2015;100:395-7.

30. Vitzthum VJ, Spielvogel $H$, Thornburg J. Interpopulational differences in progesterone levels during conception and implantation in humans. Proc Natl Acad Sci U S A. 2004;101:1443-8.

31. Nyberg CH. Diurnal cortisol rhythms in Tsimane' Amazonian foragers: new insights into ecological HPA axis research. Psychoneuroendocrinology. 2012:37:178-90.

32. Souza-Talarico JN, Plusquellec P, Lupien SJ, Fiocco A, Suchecki D. Crosscountry differences in basal and stress-induced cortisol secretion in older adults. PLoS One. 2014;9, e105968.

33. Tennison LR, Rodgers LS, Beker D, Vorobjeva KI, Creed ET, Simonenko A. Cortisol and symptoms of psychopathology in Russian and American college students. Int J Psychol. 2010;45:165-73.

34. Vitzthum VJ. The ecology and evolutionary endocrinology of reproduction in the human female. Am J Phys Anthropol. 2009;140 Suppl 49:95-136.

35. Hajat A, Diez-Roux A, Franklin TG, Seeman T, Shrager S, Ranjit N, et al. Socioeconomic and race/ethnic differences in daily salivary cortisol profiles: the multi-ethnic study of atherosclerosis. Psychoneuroendocrinology. 2010;35:932-43. 